\title{
Towards Understanding Balancing in Exertion Games
}

\author{
David Altimira \\ Human Interface Technology \\ Laboratory New Zealand \\ University of Canterbury \\ Christchurch, New Zealand \\ david.altimiracelemin@pg.canterbury.ac.nz
}

\author{
Jenny Clarke \\ School of Sport \& Physical \\ Education \\ University of Canterbury \\ Christchurch, New Zealand \\ jenny.clarke@canterbury.ac.nz
}

\author{
Gun Lee \\ Human Interface Technology \\ Laboratory New Zealand \\ University of Canterbury \\ Christchurch, New Zealand \\ gun.lee@ canterbury.ac.nz
}

\author{
Mark Billinghurst \\ Human Interface Technology \\ Laboratory New Zealand \\ University of Canterbury \\ Christchurch, New Zealand \\ mark.billinghurst@canterbury.ac.nz
}

\begin{abstract}
Playing exertion games with others can be engaging. However, players with different physical skill levels competing against each other can experience reduced engagement because they are either not challenged enough, or challenged too much. Balancing methods can address this; however, there is only limited understanding of balancing in exertion games. In this paper, we identify two distinct dimensional balancing techniques: "internal adjustment" and "external adjustment". We report results from a study where we measured player engagement after applying these adjustments to a digital table tennis game and the traditional table tennis game, finding two disengagement factors: "unexpected physical challenges" and "unacceptable competitive advantage". Based on these factors we derived a set of exertion game design considerations. We conclude that applying digital technology to a physical game can change the required skill level to play the game, and this can affect the impact of these adjustments on player engagement. These results enhances our understanding of balancing in exertion games, supporting the benefits of playing exertion games with others.
\end{abstract}

\section{Author Keywords}

Games; exertion games; sports; game balancing;

handicapping; game adjustment

\section{ACM Classification Keywords}

H.5.2. Information Interfaces and Presentation: User Interfaces
Permission to make digital or hard copies of all or part of this work for personal or classroom use is granted without fee provided that copies are not made or distributed for profit or commercial advantage and that copies bear this notice and the full citation on the first page. Copyrights for components of this work owned by others than ACM must be honored. Abstracting with credit is permitted. To copy otherwise, or republish, to post on servers or to redistribute to lists, requires prior specific permission and/or a fee. Request permissions from Permissions@ acm.org. ACE '14, November 11 - 14 2014, Funchal, Portugal Copyright is held by the owner/author(s). Publication rights licensed to ACM ACM 978-1-4503-2945-3/14/11 . \$15.00

http://dx.doi.org/10.1145/2663806.2663838

\section{INTRODUCTION}

Human Computer Interaction (HCI) has contributed to new computer based experiences that incorporate physical activity. For example allowing joggers at different locations to run together [12]. Moreover, the latest generation of video consoles use sensors such as Microsoft's Kinect [19] that require physical effort. Thus it is important to design engaging experiences to motivate people to play these games and make people practice physical activity. By engagement we mean: the quality of user experience with technology that is characterized by challenge, aesthetic and sensory appeal, feedback, novelty, interactivity, perceived control and time, awareness, motivation, interest and affect [14].

In order to create engaging experiences, it is essential to provide the right amount of challenge to the players [12] [10] [11]. When players with different skill levels play a competitive game (e.g. table tennis), the experience might not be as engaging, as explained by Flow Theory [7] [10]. Therefore, it is desirable to adjust the game experience in order to balance the players skill levels.

To accommodate players of different skills, Mueller advocates "game balancing": game adjustments that make the exertion activity not too strenuous, yet challenging for players, to optimize engagement levels [12]. We argue that game balancing can help to provide the right amount of challenge and can facilitate a more engaging experience. In studying game balancing, Mueller focused on jogging: a parallel activity where each participant's activities are performed independently [13]. We extend this work by investigating competitive non-parallel games, like table tennis, where each player functions as an obstacle that an opponent has to overcome in pursuit of the game's goals [13]. We are interested in nonparallel games because we believe game balancing might be more necessary in this type of game as a player's performance affects the other player's performance.

This paper extends previous work where Altimira et al. [3] showed that different game adjustments can have a different impact on the game score depending on the game world played in (e.g. digital or non-digital). In this paper, we build 
on this earlier work to investigate the effect of game balancing on player engagement, which can be important for designing engaging exertion games.

The main contribution of this paper is insight into player engagement when applying balancing adjustments to exertion games. When we use digital technology to play a physical game, such as table tennis, the game challenges are often altered in comparison to the non-digital game (e.g. the traditional table tennis game) thus varying the level of skill required to play the game. Our results indicate that when this happens, game balancing should be designed differently. We conclude that when we limit the physical skills in games where a large degree of skill is necessary, this can potentially increase disengagement owing to the "unexpected physical challenges". Moreover, giving a large score advantage in games where a high level of skill is not necessary can become less engaging owing to the perception of "unacceptable competitive advantage".

This work is important because it helps understand the role of the level of skill required to play a game in game balancing. It also expands our understanding of the relation between game adjustments and player engagement in exertion games. This is important for the design of balancing adjustments for exertion games as a means of motivating people to practice physical activity.

\section{RELATED WORK ON GAME BALANCING}

In this section, we summarise prior work on game balancing based on which game world the balancing was applied to (digital or non-digital) and whether the research focused on physical or non-physical games. This highlights the opportunities for learning about game balancing from the different game worlds, and from physical and non-physical games.

\section{Balancing Digital Non-Physical Games}

Balancing can be analyzed through how the game difficulty is adjusted [1, p. 340]. In digital non-physical games, Adams [1, p. 338] showed that the player perception of difficulty is comprised of four elements: the intrinsic skill required; the stress placed on the players by time pressure; the power provided (e.g. the strength of the player's character); and the amount of in-game experience (actual player skills). These elements can help game designers to understand how to challenge the players and thus balance non-physical digital games.

Prior work on digital non-physical games [1, p. 324] defined a well-balanced game as one where the players perceive the game as fair, or where the more skilled players are rewarded. Bateman et al. [5] also explained the properties of balancing methods that can cause disengagement, such as changing the gameplay or calculating the adjustments incorrectly. However, little is known about the interrelationship between game adjustments and player engagement.

\section{Balancing Digital Physical Games (Exertion Games)}

Adjusting the difficulty level in physical games can be different from non-physical games because in physical games there are additional challenges that players have to face, such as the physical effort and skills required [10]. The motivation to engage in physical games and sports can also be different to non-physical games; therefore, the strategies for game balancing and their effect on player engagement can also be expected to be different.

Prior work in balancing exertion games (digital physical games) used the players' physical exertion to balance the game [12] [17]. For example, Mueller et al. measured the physical effort exerted by joggers by taking the current heart rate of the participants and their self-determined target heart rate [12]. The difference was used to balance joggers of different fitness levels. Similarly, Stach et al. [17] used a heart rate scaling mechanism where the performance of the players was based on their effort relative to their fitness level. To understand player experience when balancing adjustments are applied, Gerling et al. [9] used a dancing game to be played in sedentary and motion-based control conditions to balance an able bodied player against a player in a wheelchair. They used hidden balancing techniques such as applying a score multiplier or time balancing; and explicit balancing techniques such as input balancing using a different step chart. They showed that while explicit adjustments can reduce selfesteem and the feeling of relatedness, hidden balancing can improve self-esteem [9]. Thus, prior work in exertion games balancing showed how physical exertion can be used to balance a game and that different game adjustments can influence the player experience differently. However, our understanding of the influence of game adjustments on player engagement is still incomplete. This understanding is important when designing engaging balanced exertion games.

\section{Balancing Non-Digital Physical Games (Traditional Sports)}

In non-digital exertion games, such as traditional sports (e.g. basketball or soccer), the balancing can be similar to that applied in exertion games. However, in non-digital physical games there are less opportunities for balancing than in digital games because there is no virtual space in which to apply the balancing adjustments. For example, in traditional sports balancing, performance is most often measured and the score adjusted [12]. There are also other ways to adjust the challenge and to provide a more balanced game, such as modifying the size of dimensions of the playing area (e.g. soccer field), presence or attitude of an audience [10, p. 46], or limiting the skills of the more skilled players (e.g. playing with the non-dominant hand in table tennis). Prior work showed us different ways that we can adjust a non-digital physical game. However, what is still missing is an understanding of the differences in balancing between traditional sports and exertion games.

\section{Player Engagement in Response to Game Balancing}

Previous studies showed that game balancing can make the game more engaging for players with different skill levels (e.g. [5]). However designing game balancing adjustments is not easy. For example, the way game balancing is designed can affect the players' self-esteem and the feeling of relatedness of the players [9]. Moreover, a game adjustment that is 
not well designed might be perceived as giving an unfair advantage to one participant over the other. Gardner argues that people might reject or accept this advantage depending on the circumstances and the way that the advantage is gained [8]. If a player gains an advantage because he or she has access to better facilities this might be perceived as acceptable. However, it might be unacceptable to use a modified bat in baseball or altered ball in golf. Gerling et al. argue that skill differences as a result of practice are seen as acceptable, but differences in physical abilities as a result of a disability that lead to different in-game scores are not [9]. Rollings et al. $[16$, p. 286] also argue that in order to make a game engaging players should perceive that the game is fair. Therefore, it is important to take into account how players perceive the advantage given to a player because it can affect the player engagement.

Changing the rules to balance a game might change how difficult it is to overcome the obstacles in pursuit of the goal, or it can even change the type of obstacles that players have to face. In both cases, a player's engagement might depend on how well the degree of difficulty matches his or her skills. Moreover, new challenges should not change what the sport was originally intended to test (e.g. the "ball-throwing" skill in bowling) [8]; otherwise the game adjustments might disengage the players. Similarly, Bateman argues that changing the gameplay in digital games can potentially decrease engagement [5]. Therefore introducing new challenges might affect the player engagement.

To summarize, prior research has shown a number of different ways we can adjust a game and how this can influence the player engagement, such as the ability to provide the right amount of challenge [7], how the adjustment is designed (e.g. explicit or implicit) [9], the acceptance of the advantage [8] [9] and the perception of fairness [16, p. 286].

\section{Design Dimensions}

Based on prior work, we determined the possible game adjustments that can be applied to balance exertion games. This helped us to decide the game adjustments to be used in this study. We classified game adjustments into two dimensions based on the elements that determine the performance outcome in sports $[4$, p.106] $[2$, p.6] $[6$, p.16] and include other elements that might influence the perception of difficulty (see Figure 1). We named these two dimensions "internal" and "external" adjustments borrowing from Weiner's model [4, p.108]. This extends the adjustment balancing dimensions of Mueller et al. [12] by explaining the different approaches of adjusting an exertion game.

The internal adjustment dimension encompasses those balancing adjustments that are applied within a player in order to balance a game. These include adjusting a player's physical skills, tactical knowledge, strategic skills, endurance, flexibility, physical endowment, fitness, experience, and emotional or psychological factors such as mood, motivations, anxiety or confidence. We were inspired by the internal factors that determine performance when practising a physical activity [2, p.6] [4, p.108] and the examples of constraints used in game sense that determine the player's perception of challenges $[6$,

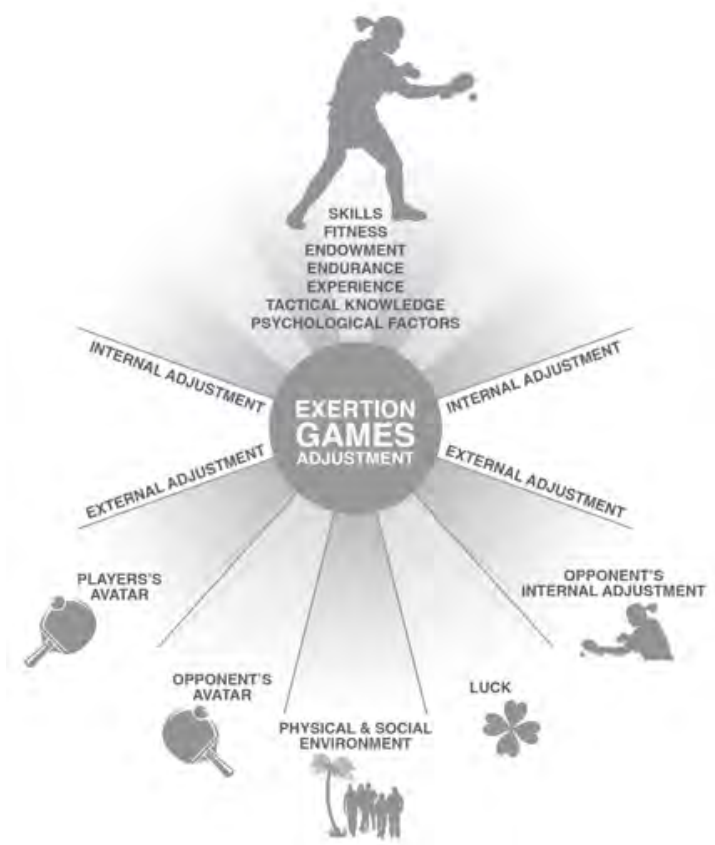

Figure 1. A two dimensional space of the available exertion game adjustments for game balancing based on an analysis of prior work

p.16]. An example of internal body adjustment is running an athletic course carrying extra weight.

The external adjustment dimension encompasses those balancing adjustments that are applied externally to a player in order to balance a game. These include adjusting the physical environment (e.g. gravity, temperature, wind, humidity) or social environment (e.g. spectators' attitudes); the task (e.g. game rules, equipment such as racket size, score/time); the opponent's skills; the power of each player's avatar (in the case players are represented by avatars), or luck. To define the external body adjustment dimension we were inspired by the work of Jackson et al. [10, p. 46] that defines the different challenges and game adjustments in sports; the work of Adams [1, p. 338] that defines the elements that compose the perception of difficulty in digital games; examples of environmental and task constraints used in game sense that determine the player's perception of challenge [6, p.16]; and Weiner's model [4, p.108] that explains the external causes of performance outcome in sports (e.g. luck, task difficulty, opponents' performance). An example of an external adjustment is to is to give a score advantage to the less skilled player.

\section{RESEARCH QUESTIONS}

Based on prior work we identified several research gaps: there is still limited understanding of the differences of game balancing between traditional sports and exertion games; and there is still a need to understand the relationship between exertion game adjustments and player engagement. Therefore, we propose the following research questions:

- R1: Do different game adjustments impact differently on the player engagement? 
- R2: Do different game adjustments impact differently on the engagement of the more skilled players, who play with disadvantage, and the less skilled players, who play with advantage?

- R3: Do game adjustments have a different impact on player engagement in traditional sports (non-digital physical games) and exertion games (digital physical games)?

- R4: Is there an interaction effect among the different game adjustments, game world played (digital or non-digital) and the player skill status (the more skilled or less skilled player)?

- R5: In what way do the different game adjustments impact on player engagement in traditional sports and exertion games?

\section{METHODOLOGY}

\section{The Game}

We chose to study table tennis as a non-parallel game because it enabled us to study the impact of game adjustments on individual players. To investigate game balancing in traditional sport and exertion games we used the traditional table tennis and the Wii Sports Resort digital table tennis game [18] (see Figure 2).

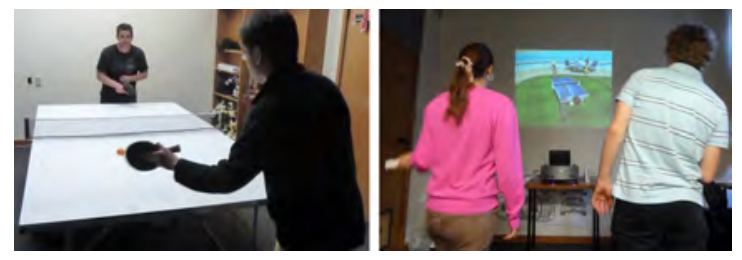

Figure 2. Traditional table tennis game setup (left) and digital table tennis game setup (right)

\section{Design of the Study}

To answer the research questions, we defined a $3 \times 2 \times 2$ mixed design with the perception of engagement as a dependent variable, and the following independent variables: game adjustment, player skill status (more skilled or less skilled) and game world (digital or non-digital, e.g. traditional table tennis game).

We defined the game adjustment as a within-subject factor with three levels: no adjustment, score adjustment and performance adjustment. We asked the players to play an 11-point game in each table tennis match. So for the score adjustment we gave a six point advantage to the less skilled player because this is the rounded average of the possible score adjustments that could be used in an eleven point game. We believed this score adjustment could be representative, taking into account the variability of the players' skill levels. For the performance adjustment we asked the more skilled players to play with their non-dominant hand. We chose these two game adjustments as two examples of the available exertion game adjustments (see section "Design Dimensions") because they can be applied to both digital and traditional table tennis games and balance the chances of winning of the players and thus provide the right amount of game challenge.
Although we acknowledge that explicit game adjustments are less desirable than implicit ones because they can impact the players' experience more negatively [9], game adjustments are often difficult to hide. This is often the case when balancing non-digital games. That is why we decided to apply explicit game adjustments for our study. We also acknowledge that each of these game adjustments proposed might be more suitable in one game world than the other. However, our aim was to understand the differences between applying game adjustments in these two game worlds. The order in which we imposed these three conditions was counterbalanced to avoid any order effect.

We defined the player skill status and the game world to be played as between subject factors. In every match one player was assigned as "the more skilled player of the match", and the other as "the less skilled player of the match". This was determined by assessing each player's skills using a prequestionnaire prior to the main experiment (see section "Players"). We chose to use a questionnaire because this allowed us to pair the players with different skill levels and assign them to play the traditional table tennis game or the digital table tennis game prior to the main experiment. The player's skill status determined to whom we applied the advantage and disadvantage in the different game adjustment conditions.

\section{Players}

For this study we selected a sample of the population aged $18+$ that have previously played the traditional table tennis or exertion games such as Wii sports games. We recruited 46 players, mainly from the local university, 37 males and 9 females, whose ages ranged from 19 to 43 years, with a mean of $M=26.7$ and $S D=4.9$. Each player completed an online pre-experiment questionnaire in which we asked them to rate their skill level [0: low skill level to 100: high skill level] and their frequency of playing (never, less than once a month, once a month, $2-3$ times a month, once a week, $2-3$ times a week, daily) with the traditional table tennis game and the Wii table tennis game. We also asked about the frequency of playing other exertion games such as other Wii sports games in case they were not familiar with our digital test game. We assigned the players to the digital or non-digital game based on the information from the pre-questionnaire with the following objective: create pairs of players with as large a difference in skill level as possible between the players of each pair in each game world. Sixteen players were assigned to play the digital game and 30 players to the traditional table tennis game.

For the players assigned to play the traditional table tennis game, the self-reported skill level of the players that were grouped as the more skilled players had a $M=66.89$ and $S D=17.02$. In contrast, the players grouped as the less skilled players had a $M=33.73$ and $S D=18.88$. Moreover, the Fisher's exact test showed the more skilled players tended to play table tennis significantly more frequently than the less skilled players $(p=.03)$.

For the players assigned to play the digital table tennis game, the self-reported skill level of the players with our digital test game was not as informative because the players tended to 
rate their Wii table tennis skill level quite low $(M=26.92$ and $S D=24.72$ ). The players seemed to be quite unfamiliar with our digital test game, and therefore we decided to separate players into skilled and non-skilled based on their frequency of play with other exertion games such as Wii sport games. We believed the familiarity of players with Wii mote input device (the tool players used to play our digital test game) could provide a competitive advantage over those players unfamiliar with this device. The Fisher's exact test showed the players grouped as the more skilled tended to play exertion games such as other Wii sport games significantly more frequently than those players grouped as the less skilled players $(p=.01)$.

\section{Procedure}

Each pair of players played for five minutes to warm-up. Then we asked them to play a competitive 11-point game in each game condition. After playing in each game condition we asked each player to complete a questionnaire assessing his or her perception of engagement. Afterwards, we interviewed the players individually following a semi-structured interview.

\section{Data Analysis}

We collected both quantitative and qualitative measures. The quantitative analysis was performed to answer the research questions RQ1-RQ4 using a repeated measures ANOVA with the game adjustment condition as a within factor, and the player skill status and the game world played as between factors. The qualitative feedback was analyzed to answer RQ5, which allowed us to understand the reasons for the reported levels of engagement. For the qualitative analysis we used the players' report from the voice-recorded semi-structured interviews and observations of players playing the digital game and the traditional table tennis game in each of the game adjustments.

To collect information about the experience of players in each condition we used the engagement questionnaire from the O'Brien model of engagement [15], which we adapted to the gaming context (e.g. changing the statement "The time I spent shopping just slipped away" to "The time I spent playing the game just slipped away"). This model of engagement (engagement with technology) is not tied to the videogames context and therefore we believed it was suitable for the study. The engagement score is obtained based on the user's rating of several different factors: focus of attention, perceived usability, aesthetics, endurability, novelty and the involvement felt. In this study we excluded the aesthetic factor as it was not relevant to the traditional table tennis game.

\section{RESULTS}

\section{Engagement}

The results of the engagement score (means and S.E.) are shown in Figures 3, 4 and 5. We now discuss the results in response to our research questions:

R1: There were no significant differences in engagement among the three game adjustment conditions $(F(2,32)=$ $0.24, p=.79)$.

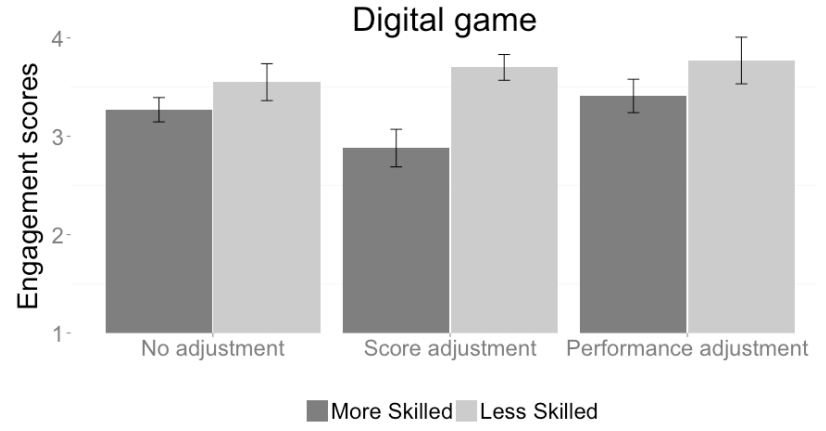

Figure 3. Engagement scores [1-5] of the more skilled and less skilled players playing the digital table tennis with no adjustment, score adjustment and performance adjustment.

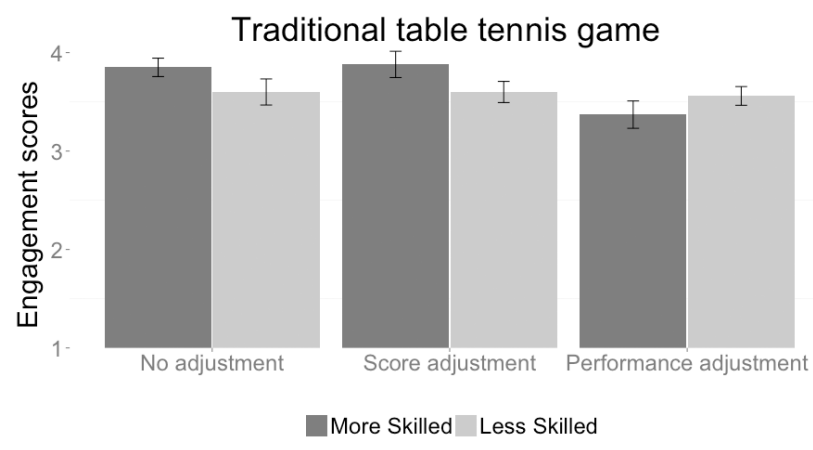

Figure 4. Engagement scores [1-5] of the more skilled and less skilled players playing traditional table tennis with no adjustment, score adjustment and performance adjustment.

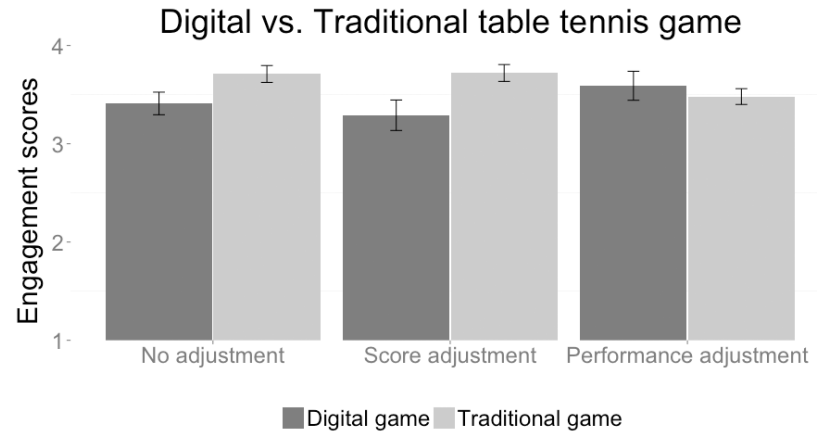

Figure 5. Engagement scores [1-5] of the players playing traditional table tennis and digital table tennis with with no adjustment, score adjustment and performance adjustment

$\mathrm{R} 2$ : There was no interaction effect between the game adjustment and the player skill status $(F(2,32)=1.27, p=.30)$.

R3: In the digital table tennis game players reported lower engagement than in the traditional table tennis game in the noadjustment and score adjustment conditions (see Figure 5). However, this tendency was reversed in the performance adjustment condition where players in the traditional table tennis game experienced a decrease in the engagement, while those in the digital game reported an increase in engagement (see Figure 5). This change of tendency in the performance adjustment condition was significant as it was shown in the interaction effect analysis between the game adjustment and the game world played $(F(2,32)=5.06, p=.01)$. 
R4: The engagement scores for the less skilled players, who were advantaged by the game adjustments applied, did not seem to change significantly among the game adjustments in both digital and traditional table tennis games (see Figure 3 and 4). The range of the players' engagement scores in the digital game ratings was $[3.55,3.77]$ and in the traditional table tennis it was [3.56, 3.60]. However the experience for the more skilled players was different. The range of the players' engagement scores in the digital game was [2.88, 3.41] and in the traditional table tennis it was [3.37, 3.88]. In the traditional table tennis game the scores in the no-adjustment and in the score adjustment conditions were similar, but they dropped in the performance adjustment condition (see Figure 4). Similarly, in the digital game, the scores in the noadjustment and performance adjustment conditions were also similar but they decreased in the score adjustment condition (see Figure 3). This means the game world played influenced how the adjustments impacted the engagement scores of the more skilled players. That is why we found an interaction effect of the game adjustments, game world played and the player skill status $(F(2,32)=4.45, p=.02)$.

R5: The two most frequently reported factors that the game adjustments affected were the perception of challenge and the perception of unfairness.

\section{The Perception of Challenge}

According to the interviews, the less skilled players reported a less challenging experience when playing with a game adjustment. However, the more skilled players (38\% of those playing in the digital game and $56 \%$ of those in the traditional table tennis game) reported that the score adjustment increased their amount of concentration to get points faster. Moreover, the more skilled players (50\% of those playing in the digital game and $78 \%$ of those playing in the traditional table tennis game) also pointed out that playing with the nondominant hand changed their game strategies because they had to focus on controlling the table tennis racket or Wii controller. For example, one player playing in the traditional table tennis game reported: "I need to be more careful in the game (...). I have to think more when I move my hand to actually (...) you know (...) calculate where I should place my hand (...)". Of the players in the traditional table tennis game playing with the non-dominant hand, $44 \%$ went further, stating that this adjustment changed their game goals.

Based on our observations of the players playing with the non-dominant hand in the traditional table tennis game and the number of mistakes we noted players making, we concluded that players felt quite uncomfortable owing to the lack of sense of control. To summarize, for the more skilled players, the score and performance adjustments impacted the players' challenges differently and the strength of the impact seemed to be higher in traditional table tennis game than in the digital game.

\section{The Perception of Unfairness}

From the players' reports, the players' engagement scores were influenced by their perception of unfairness of the different game adjustments. For example, one player, who played with a score advantage in the traditional table tennis game, answered the following when asked about which was the preferred condition: "The fair one, the no handicap (...) I felt bad I won because of the handicap, it was not very satisfying". Another player who played with a disadvantage in the digital table tennis game answered the following when asked about why the game adjustments provided a less engaging experience although providing a higher amount of challenge: "Because it was not fair, so (...) in that case, I did not want to play. If it is not fair, I do not want to play, I do not want to enjoy the game".

\section{DISCUSSION}

Using digital technology to play a physical game such as table tennis often simplifies the player-game interaction in comparison to the non-digital game (e.g. the traditional table tennis game). The degree of simplification might depend on several factors, such as the design of the game, how the technology is implemented, and the accuracy of the sensors used. The two test games used in our study (i.e. the traditional table tennis game and a digital table tennis game) are clear examples of how digital technology can lower the accuracy required by the players and how it can adjust the level of skill required to play the game. We argue that when this happens, game balancing should be different. For example, our previous work showed that playing with the non-dominant hand affected the score of the players playing in the traditional game more than those who played the digital game [3]. Therefore, altering the level of skill required to play a game can affect the effectiveness of game adjustments (e.g. to balance the score). We show that it can also affect the impact of game adjustments on player engagement.

The influence of game adjustments on player engagement was different between the more skilled player, who played with a disadvantage, and the less skilled player, who played with an advantage. While the less skilled players did not report significant changes in engagement among the conditions in any of the game worlds played, the more skilled players tended to be more disengaged in the score adjustment than the other game adjustment conditions in the digital game. Similarly, the more skilled players tended to be more disengaged in the performance adjustment than the other game adjustments conditions in the traditional table tennis game.

From the observations of players playing, we hypothesize that the more skilled players might have played slightly more sportingly in the no-adjustment condition. This might have reduced the impact the more skilled players had on their opponent's performance and the impact the different skill levels between players had on the less skilled player's engagement. This might account for the results of engagement for the less skilled players.

Regarding the more skilled players' disengagement, we used the results of the interviews and direct observations during the play to derive two key factors of disengagement that we named "unexpected physical challenges" and "unacceptable competitive advantage". 


\section{Disengagement Factor 1: Unexpected Physical Chal- lenges}

The more skilled players tended to disengage when playing with the non-dominant hand in traditional table tennis. Playing with the non-dominant hand changed the player's game goals and strategies (see section The Perception of Challenge) because it increased the physical challenge of manoeuvring the table tennis bat. Players were not used to playing table tennis with this type of physical challenge. As a result, players experienced a decreased sense of control, which made the game frustrating, leading to disengagement. However, in the digital game, players using the non-dominant hand did not experience decreased engagement. We believe the reason is that the digital game required a lower skill level to play and therefore playing with the non-dominant hand did not affect the players as much as in the traditional game.

Design consideration: Increasing the required skill level to play can increase the impact that a performance adjustment has on the players. In this scenario the game adjustment can introduce an unexpected physical challenge, which game designers should be wary of as in some cases it can lead to player disengagement.

\section{Disengagement Factor 2: Unacceptable Competitive Ad- vantage}

The more skilled players tended to disengage when playing with a score disadvantage in the digital table tennis game. We believe this is because the players did not accept the disadvantage (see section "Engagement"). However, the more skilled players did not disengage with the same score adjustment in the traditional table tennis. We believe this is because in the traditional game these players had a greater possibility to win with this adjustment (see [3]). The digital game required a lower skill level to play and therefore the performance of the two players was more similar in the digital game than in the traditional table tennis game. Gerling et al. previously pointed out that overbalancing might affect the experience of the stronger player [9]. Finally, these results align with Gardner who claimed that the perception of fairness of a game adjustment can be different depending on the circumstances [8].

Design consideration: Lowering the required skill level to play a game can lessen the difference in game performance between players. Therefore, we suggest that a lower score adjustment should be applied to games that require a lower skill level to play to avoid overbalancing the game and thereby increasing the chance of disengagement owing to the unacceptable competitive advantage.

\section{The Design of Static Game Adjustments}

Our results highlight two potential risks of the design of static game adjustments: these are adjustments made at the start of the game that remain unchanged for the duration of the game. First, if the adjustment fails to balance the game (i.e. does not make enough difference to give the less skilled player a chance to win, or overbalances to give the less skilled player too much of an advantage) then the effect on engagement can be worse than when making no adjustment at all. This is what happened with the score adjustment in the digital table tennis game. Second, if players lose a sense of control as a result of the adjustment (e.g. playing with the non-dominant hand) and the game does not give the player the chance to take any decision or action to overcome this new challenge, the game can then become frustrating, leading to disengagement. It is important to take these reflections into account when designing balancing adjustments to produce more engaging games.

\section{Generalization of Findings}

Our primary contribution in this work is providing insight into the role that the level of skill required in a game has on the player engagement when balancing exertion games. We have provided evidence that game balancing should be different in games that require different skill levels to play. Since the focus is on the level of skills required in a game, the findings are applicable to other exertion games beyond those studied in the present work.

Our findings are also relevant to non-physical games. For example, the relation between the player's skills needed to play a game, the score adjustment, and the non-acceptance of the competitive advantage could also be expected in other nonphysical games. The disengagement owing to the unexpected physical challenges when limiting the physical skills might be mainly relevant to physical games as physical skills are more a characteristic of physical than non-physical games. However, it is expected that in non-physical games there are other adjustments that can create unexpected challenges, leading the players to disengage.

For this research we studied a non-parallel game (i.e. a table tennis game) where each player's performance affects their opponent's performance. Although we chose a non-parallel game, we believe our findings are relevant to parallel games because these findings do not seem to depend much on the non-parallel aspect of the games. However, the study of game adjustments in parallel games is left as future work.

\section{Limitations of this Study}

We did not have much control over the internal mechanics of the digital game since we used an existing digital game, i.e. Wii Sports Resort [18]. Although we were not able to confirm whether it has an internal balancing method implemented, it appears it is very unlikely to have such feature by observing how challenging it was for the more skilled players to catch up in the score adjustment condition.

We assessed the player's skills using a pre-questionnaire. As reported in section "Design of the Study", this was useful because it allowed us to pair the players prior to the main experiment. Although this method of assessing the players' skills was enough for the purpose of this study, we acknowledge that we might have obtained a more accurate assessment of the players' skills by observing them playing before the main experiment. Finally, the limited number of players meant we could not perform an analysis of whether (and how) the motivation of the players to practise physical activity influenced the engagement scores when playing with our proposed game adjustments. 


\section{CONCLUSIONS}

We reported results from a study where we measured and investigated the player engagement after applying exertion game adjustments to a digital table tennis game and the traditional tablet tennis game in an attempt to balance the win probabilities of players with different skill levels. This work provides insight into player engagement when applying these adjustments.

The use of digital technology to play physical games can alter the level of skill required to play the game in comparison with the non-digital game when the digital game requires a lower player accuracy to perform a game action. The two table tennis games we studied showed clear examples of this. We argue that when this happens, game balancing should be different. For example a six point adjustment in an eleven point game can be more suitable in the non-digital table tennis game than in the digital one. Our primary contribution here is insight into the role that the level of skill required in a game has on the player engagement when balancing exertion games. We identified two factors of disengagement and for each factor we proposed game design considerations.

We have explored two different possible game adjustments in exertion games, and this work enhances our understanding of balancing exertion games. For future research direction, we will study still unexplored game adjustments such as adjusting the luck, the physical or the social environment (see section "Design Dimensions"), and their impact on player engagement. In this study we investigated digital and nondigital games. We believe it would be also interesting to study mixed-reality games in future work, and explore, for example, how to use digital technology to balance a physical game in order to enhance the player's engagement. Moreover, we would like to extend this work by applying it to other nonparallel exertion games such as Squash or Badminton. Understanding each of the available adjustments for balancing exertion games and their influence on player engagement will help creating more engaging exertion games. As a result, this can motivate more people to practice physical activity and thereby enjoy the many benefits of doing so.

\section{ACKNOWLEDGMENTS}

We thank all the volunteers who participated in this study and helped in making this work possible and Tim Ryan for the illustrations.

\section{REFERENCES}

1. Adams, E. Fundamentals of game design. New Riders, Berkeley, Calif., 2010.

2. Alderman, R. B. Psychological behavior in sport. Saunders, Philadelphia, 1974.

3. Altimira, D., Billinghurst, M., and Mueller, F. Understanding handicapping for balancing exertion games. In CHI'13 Extended Abstracts on Human Factors in Computing Systems, CHI EA'13, ACM (New York, NY, USA, 2013), 1125-1130.

4. Anshel, M. H. Sport psychology: from theory to practice. B. Cummings, San Francisco, 2012.

5. Bateman, S., Mandryk, R. L., Stach, T., and Gutwin, C. Target assistance for subtly balancing competitive play.
In Proceedings of the SIGCHI Conference on Human Factors in Computing Systems, CHI '11, ACM (New York, NY, USA, 2011), 2355-2364.

6. Breed, Ray., S. M. Developing game sense through tactical learning : a resource for teachers and coaches. Cambridge University Press, Cambridge ; Port Melbourne, Vic., 2011.

7. Csikszentmihalyi, M. Flow: the psychology of optimal experience. Harper \& Row, New York, 1990.

8. Gardner, R. On performance-enhancing substances and the unfair advantage argument. Journal of the Philosophy of Sport 16, 1 (1989), 59-73.

9. Gerling, K. M., Miller, M., Mandryk, R. L., Birk, M. V., and Smeddinck, J. D. Effects of balancing for physical abilities on player performance, experience and self-esteem in exergames. In Proceedings of the SIGCHI Conference on Human Factors in Computing Systems, CHI '14, ACM (New York, NY, USA, 2014), 2201-2210.

10. Jackson, S. A., and Csikszentmihalyi, M. Flow in sports. Human Kinetics, Champaign, IL, 1999.

11. Kretchmar, R. S. Practical philosophy of sport and physical activity. Human Kinetics, Champaign, IL, 2005.

12. Mueller, F., Vetere, F., Gibbs, M., Edge, D., Agamanolis, S., Sheridan, J., and Heer, J. Balancing exertion experiences. In Proceedings of the SIGCHI Conference on Human Factors in Computing Systems, CHI '12, ACM (New York, NY, USA, 2012), 1853-1862.

13. Mueller, F. F., Gibbs, M. R., and Vetere, F. Taxonomy of exertion games. In Proceedings of the 20th Australasian Conference on Computer-Human Interaction: Designing for Habitus and Habitat, OZCHI '08, ACM (New York, NY, USA, 2008), 263-266.

14. O'Brien, H. L., and Toms, E. G. What is user engagement? a conceptual framework for defining user engagement with technology. J. Am. Soc. Inf. Sci. Technol. 59, 6 (Apr. 2008), 938-955.

15. O'Brien, H. L., and Toms, E. G. The development and evaluation of a survey to measure user engagement. $J$. Am. Soc. Inf. Sci. Technol. 61, 1 (Jan. 2010), 50-69.

16. Rollings, Andrew, A. E. Andrew Rollings and Ernest Adams on game design. New Riders, Indianapolis, Ind., 2003.

17. Stach, T., Graham, T. C. N., Yim, J., and Rhodes, R. E. Heart rate control of exercise video games. In Proceedings of Graphics Interface 2009, GI '09, Canadian Information Processing Society (Toronto, Ont., Canada, Canada, 2009), 125-132.

18. Wii Sports Resort. http://www.wiisportsresort.com/.

19. XBox Kinect. http://www.xbox.com/en-GB/kinect. 